\title{
Chronopharmacology of Anti-Convulsive Therapy
}

\author{
Sriram Ramgopal • Sigride Thome-Souza • \\ Tobias Loddenkemper
}

Published online: 3 March 2013

(C) The Author(s) 2013. This article is published with open access at Springerlink.com

\begin{abstract}
Approximately one-third of patients with epilepsy continue to have seizures despite antiepileptic therapy. Many seizures occur in diurnal, sleep/wake, circadian, or even monthly patterns. The relationship between biomarkers and state changes is still being investigated, but early results suggest that some of these patterns may be related to endogenous circadian patterns whereas others may be related to wakefulness and sleep or both. Chronotherapy, the application of treatment at times of greatest seizure susceptibility, is a technique that may optimize seizure control in selected patients. It may be used in the form of differential dosing, as preparations designed to deliver sustained or pulsatile drug delivery or in the form of 'zeitgebers' that shift endogenous rhythms. Early trials in epilepsy suggest that chronopharmacology may provide improved seizure control compared with conventional treatment in some patients. The present article reviews chronopharmacology in the treatment of epilepsy as well as future treatment avenues.
\end{abstract}

Keywords Epilepsy · Chronotherapy ·

Chronopharmacology $\cdot$ Chronopharmacokinetics .

Differential dosing $\cdot$ Seizures $\cdot$ Seizure patterns $\cdot$ Diurnal

This article is part of the Topical Collection on Sleep

S. Ramgopal $\cdot \mathrm{S}$. Thome-Souza $\cdot \mathrm{T}$. Loddenkemper $(\triangle)$

Division of Epilepsy and Clinical Neurophysiology,

Harvard Medical School, Fegan 9,

Boston, MA, USA

e-mail: tobias.loddenkemper@childrens.harvard.edu

S. Ramgopal $\cdot$ S. Thome-Souza $\cdot$ T. Loddenkemper

Department of Neurology, Children's Hospital Boston, 300

Longwood Ave,

Boston, MA 02115, USA

\section{S. Thome-Souza}

Psychiatry Department of Clinics Hospital of Faculty of Medicine,

University of Sao Paulo,

Sao Paulo, Brazil patterns $\cdot$ Closed loops $\cdot$ Melatonin $\cdot$ Light therapy $\cdot$ Dim light melatonin onset $\cdot$ DLMO $\cdot$ Circadian rhythms $\cdot$ Circadian pattern $\cdot$ Suprachiasmatic nucleus $\cdot$ Seizure tracking $\cdot$ Seizure diary $\cdot$ Chronopharmacodynamics $\cdot$ Pharmacokinetics · Pharmacodynamics

\section{Introduction}

Epilepsy affects nearly $1 \%$ of the population. In spite of the development of a greater number of medications over the last decade, nearly one-third of epilepsy patients continue to have seizures despite available pharmacological approaches [1]. Only some patients qualify for epilepsy surgery [2] or may be treated with VNS, deep brain stimulation, or ketogenic diets $[3,4]$. Therefore, there is an urgent medical need to improve epilepsy management to provide improved seizure control. A therapeutic intervention that consists of treatment timing at times of greatest seizure susceptibility may help some patients that do not respond to conventional pharmacotherapy. Additional evidence on seizure patterns throughout day and night is becoming available, and a relationship between seizure timing and the localization and semiology of seizures has been suggested $[5 \cdot, 6,7 \cdot]$. With the aid of seizure prediction models and the identification of periods of greater seizure risk, additional strategies may be developed to provide improved seizure control at times when seizures are most likely. Few studies have evaluated chronotherapy in the treatment of epilepsy $[8 \bullet \bullet, 9 \bullet \cdot]$. We provide an overview of chronotherapy, diurnal and nocturnal patterns of seizure clustering, and preliminary applications demonstrating the use of chronotherapy in the treatment of epilepsy.

Ways to Assess Timing and Biological Rhythms

While 24-hour clock times and sleep/wake rhythms frequently overlap with the internal clock, they do not always 
match the circadian rhythm. There are a variety of methods to ascertain the timing of biological clocks. Melatonin provides the most reliable and consistent measure of the circadian pattern and can be measured in the plasma, saliva, or urine [10]. Because secretion of the hormone is acutely suppressed by light exposure, the measurement of the time of onset of the daily melatonin rise during low-light exposure is a more reliable measure of the circadian phase [10, 11]. The dim-light melatonin onset (DLMO) has been used to assess alterations of circadian phase in a variety of diseases [12-14], and has also been trialed in epilepsy [15]. Other markers, such as core body temperature, and cortisol may also serve as biomarkers for circadian rhythms. Cortisol can be measured in serum and saliva. Cortisol typically rises in the late evening and early morning. Another marker, core body temperature usually falls during nighttime and rises during the morning to afternoon [10]. Body temperature can be measured through wrist and rectal measurements, though corrections are needed to account for different activities and climates [16]. Due to overlap of circadian rhythms and sleep cycle, forced separation of both can also serve as a tool to assess the effect of sleep and of the circadian rhythm separately. For that purpose, forced desynchrony paradigms separating sleep/wake cycle and circadian rhythms have been designed [17].

\section{Chronotherapeutics}

\section{Aligning Therapy to Endogenous Rhythms}

Chronopharmacology refers the "design and evaluation of drug delivery systems that release a bioactive agent at a rhythm that ideally matches the biological requirement of a given disease therapy" [18]. Chronotherapy can be delivered through a variety of strategies and may include both time and site-specific drug application regimens. Differential dosing strategies, for example, may rely on distributing the dosage of the drug to correspond with periods of maximal disease severity (Fig. 1). Other strategies may employ drug delivery systems which allow, depending on the need, for sustained or pulsatile release of drug at the location in the body where it is needed most. Zero-order release systems ensure that a constant amount of drug is transferred into the plasma over time. Similar systems and devices may be implemented in epilepsy care utilizing osmotically driven pumps and controllable swelling matrices [19•]. These systems can ensure a constant plasma level of drug. Pulsatile drug delivery systems, in which the plasma level of drug fluctuates in a periodic manner, have been formulated using microparticles, coarse particles, solid implants, hydrogels, osmotic pumps, and liposomes [19•]. Through the use of these strategies, the timing of drug availability may be optimized for a given disease with symptoms that follow a periodic pattern.
Biological Basis of Chronotherapeutics

Chronopharmacological techniques ensure that drug levels in the blood are within therapeutic ranges during periods of maximal disease severity. An example of this is seen in how evening doses of antihypertensive therapy can be used to prevent morning rises in blood pressure [20]. The evening dose of the drug may thus be well timed with diurnal changes in blood pressure, preventing diurnal worsening of hypertension.

Receptor changes and receptor holidays experienced during trough periods of drug activity may decrease tolerance to antiepileptic medications, thereby improving their efficacy during periods of greater need. The sensitivity of rats with experimental Parkinson's disease exposed to dopaminergic drugs, for example, can be temporarily enhanced following short term treatment withdrawal [21]. In addition, medications may have a different effect based on the timing of the dose. The efficacy of ketamine, for example, has been shown to have varying efficacy based on the timing of dose despite reaching equivalent plasma concentrations, giving rise to the theory that some of these diurnal effects may be due to changes in receptors or secondary messenger systems [22]. Chronotherapy may prevent up- or down-regulation of receptors during periods of lesser need allowing optimal efficacy during periods of disease exacerbation.

\section{Chronotherapy in Chronic Medical Conditions}

Chronotherapy has shown promise in a number of diseases. Asthma, for example, can be managed through timed dosing to provide superior symptom control [23]. The finding that bedtime blood pressure serves as an independent risk factor for cardiovascular events in diabetics has prompted interest in time-locked therapies to specifically reduce blood pressure during these hours [24]. Targeted treatments towards circadian rhythms may play an important role in the treatment of obesity [25]. Chronotherapy may be also useful in formulating treatments for conditions such as tumors [26], arrhythmias [27], ischemic heart disease [28], psychiatric disease [29], HIV [30], diabetes [31], and other chronic medical conditions.

\section{Seizure Patterns in Epilepsy}

Early observations of relationships by Gowers linking sleep/ wake and diurnal patterns in epilepsy can be dated to the late 1800s [32]. Additional patterns in epilepsy have been elucidated over the last few decades (Table 1). Some of these patterns have been identified in relation to the 24-hour clock, the sleep/wake cycle, and the circadian rhythm. Beyond these, other cycles may also be involved, including catamenial 
Fig. 1 Differential dosing strategies are an example of chronopharmacological treatment. The dosage of drugs can be adjusted to correspond with times of greatest disease severity or susceptibility. The figure on top (a) includes a solid line that depicts variations in plasma levels of antiepileptic medication in individuals who received a higher evening dose of carbamazepine, as ascertained by computer generated pharmacokinetic modeling (solid line). The figure below (b) illustrates the percentage distribution of frontal lobe seizures over a 24-hour period, as demonstrated in a series of 41 consecutive pediatric patients. Figure modified and adapted with permission from Guilhoto et al. 2011. [8••]
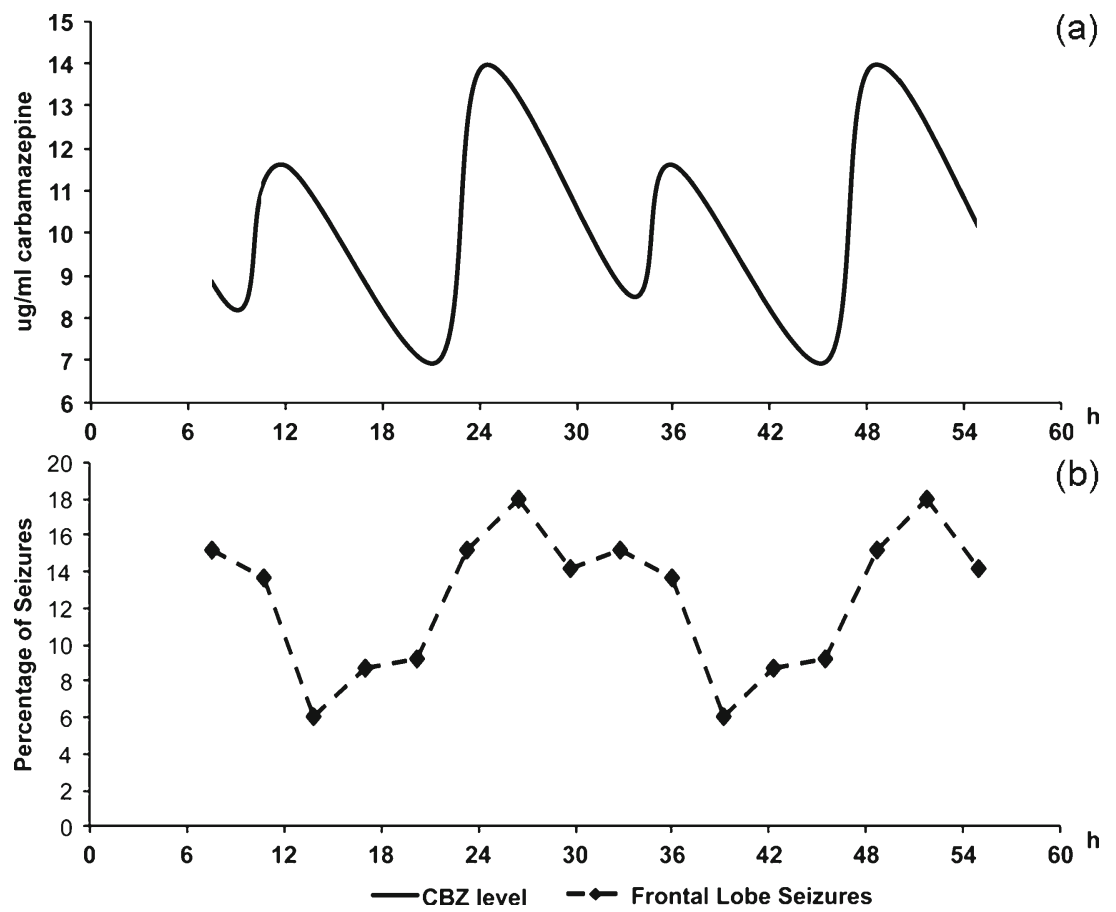

seizures. Other factors that may account for seizure patterns over larger timeframes may involve seasonal variations in climate [33, 34] and infections [35]. Epilepsy may therefore be an excellent candidate for chronopharmacological treatment approaches due to suspected associations of seizure exacerbation with some of these biological rhythms and triggers.

\section{Diurnal Patterns of Seizures}

Many seizures follow a diurnal pattern and these patterns are influenced by factors including EEG localization, seizure semiology, etiology, and age.

\section{EEG Localization}

The most consistent seizure pattern descriptions are related to ictal EEG localization. Temporal lobe seizures have been documented to occur more in the afternoon $[5 \cdot 36,38]$ and may also have an additional morning peak $[5 \cdot, 6]$. Frontal lobe seizures occur more in the early morning $[5 \bullet, 6]$. Data for occipital lobe and parietal lobe seizures are based on fewer reported patients, but occipital lobe seizures may occur more in the afternoon and parietal seizures may occur more in the morning $[5 \bullet, 6]$. Generalized seizures are documented to occur most frequently in the early morning $[6,39 \bullet]$.

\section{Semiology}

Fewer studies have investigated seizure patterns based on their semiological manifestations. However, diurnal patterns have been identified for tonic, clonic, tonicclonic, automotor, hypermotor, atonic, myoclonic, hypomotor, and dyscognitive seizures and epileptic spasms [6, $36,40]$. Secondary generalized seizures have been noted to occur more frequently at night [41]. Clinical seizure evolutions may serve as an additional factor in predicting seizure occurrence [42].

Age may affect timing of seizures, as demonstrated in a study analyzing diurnal patterns of epileptic spasms in different age groups [40] and a study on secondary generalizations [41].

\section{Sleep/Wake Patterns of Seizures}

A comparison between sleep/wake and 24-hour timing of seizures found sleep/wake status to be more predictive of seizure occurrence [6]. Sleep/wake patterns have been studied in respect to EEG localization, and seizure semiology. Other factors may play a role as well. One study, for example, noted that patients with Taylor type focal cortical dysplasia were more likely to have seizures out of sleep [43].

\section{EEG Localization}

Temporal lobe epilepsy occurs more during wakefulness, as established in a variety of adult and pediatric studies $[6,44]$. Generalized seizures classically occur soon after arousal [39•]. Occipital and parietal lobe seizures may also occur more out of wakefulness [45], and frontal lobe seizures occur more during sleep [44]. 


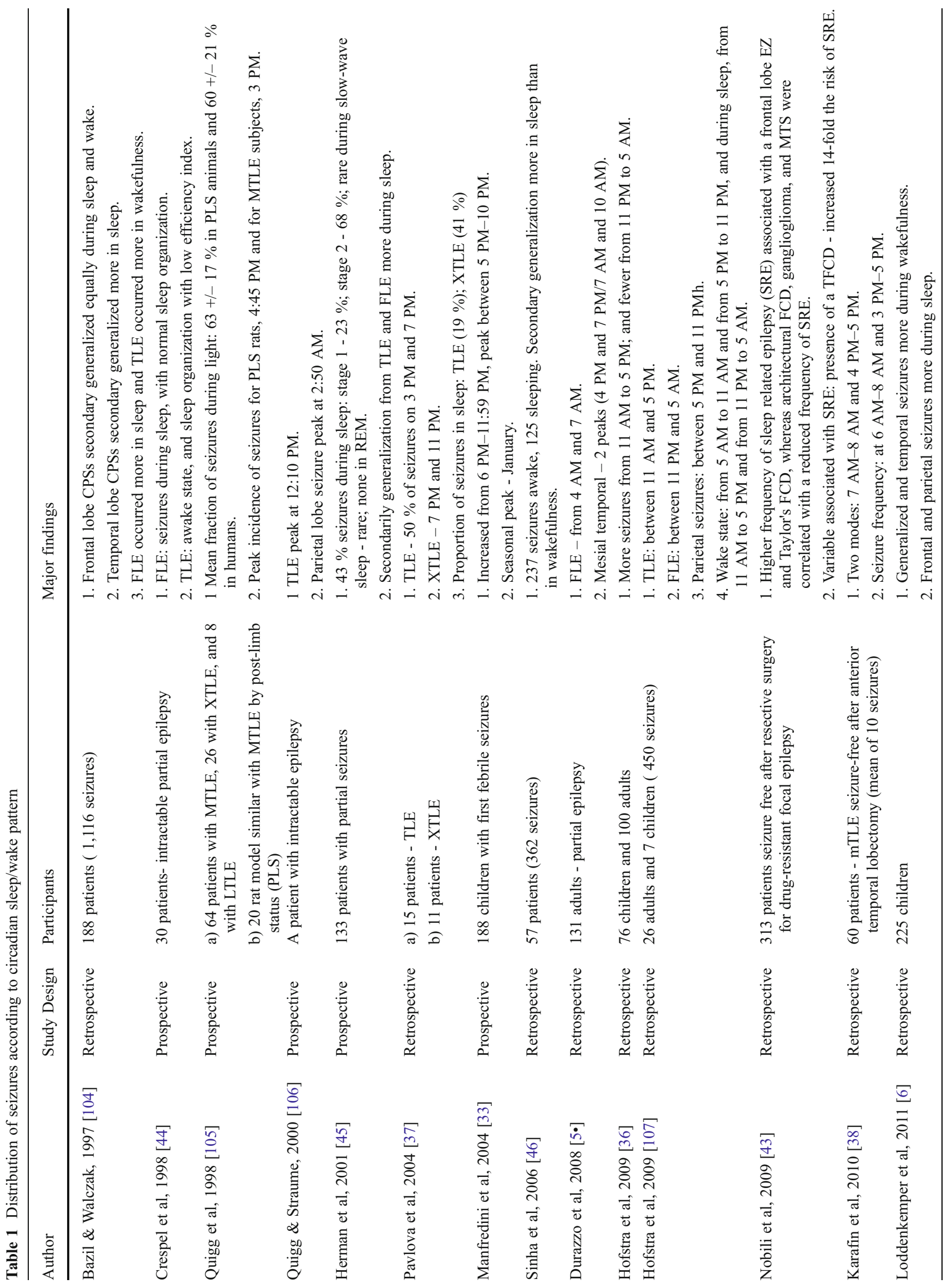




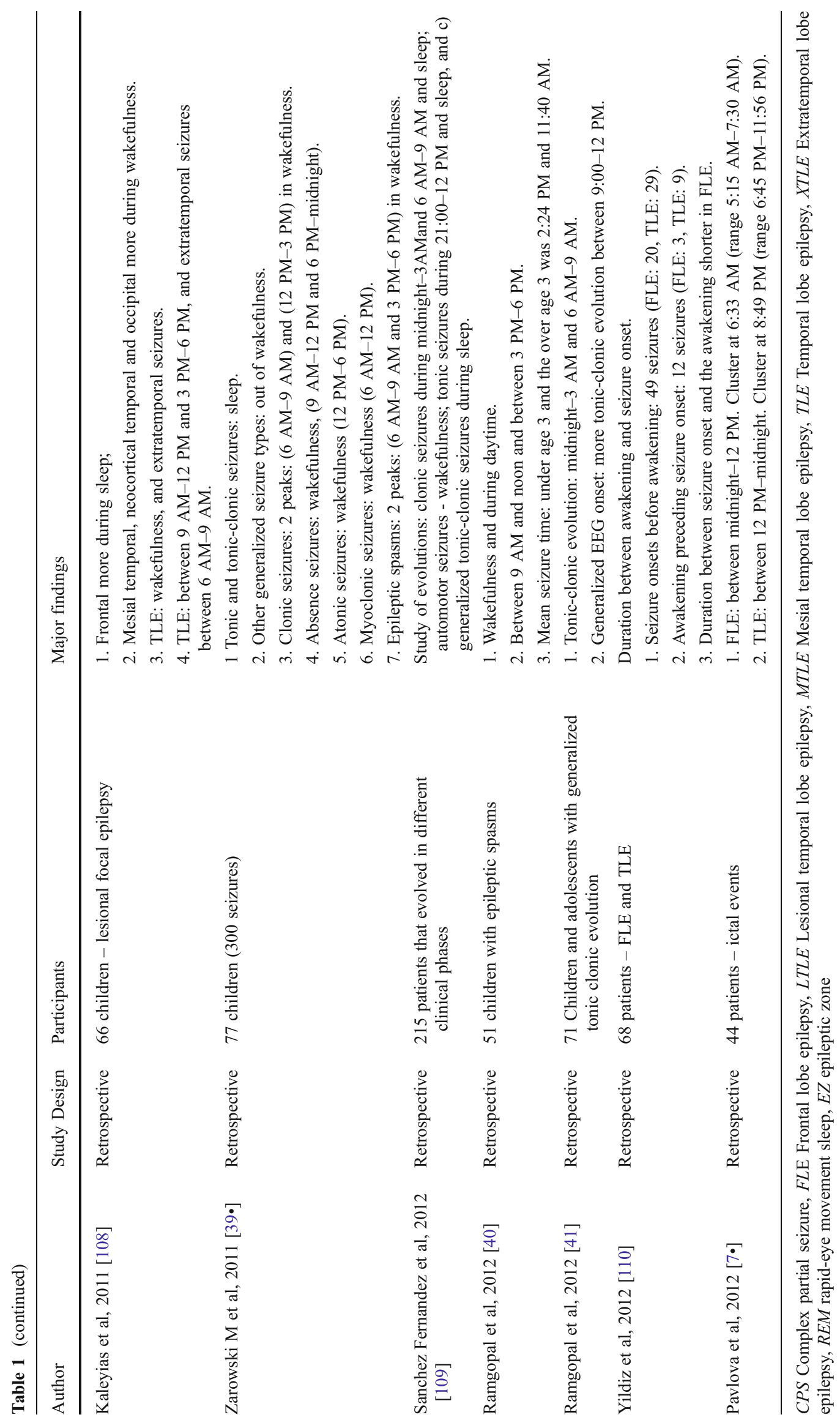




\section{Seizure Semiology}

Auras, hypomotor, atonic, dyscognitive seizures and epileptic spasms occur significantly more frequently during wakefulness. Tonic, automotor, and hypermotor seizures occur more during sleep [6]. Secondary generalized seizures occur more out of sleep, demonstrated in both pediatric [41] and adult $[45,46]$ studies.

\section{Circadian Patterns of Seizures}

A pilot study investigated associations between DLMO and the occurrence of seizures in patients with temporal and frontal lobe epilepsy undergoing video-EEG [15]. These authors found that temporal lobe seizures occurred most frequently 6 hours before the melatonin onset and frontal lobe seizures occurred most frequently 6-12 hours after this time. More research is needed to definitively associate the circadian rhythm to the occurrence of epileptic seizures.

\section{Catamenial Patterns in Seizures}

The exact patterns of catamenial seizures vary. In a study of 184 women with epilepsy, seizures clustered around the menstrual cycle in 3 patterns: a peri-menstrual pattern, an ovulatory phase pattern, and a luteal phase pattern [47]. These patterns may relate to imbalances between proconvulsant estrogen and anticonvulsant progesterone, or may occur secondary to alterations in water balance or antiepileptic drug metabolism [48]. Treating seizures with higher epilepsy medication doses at times of greatest seizure susceptibility may relieve seizure burden.

\section{Lunar Patterns in Seizures}

Changes in lunar phases have long been believed to be associated with the timing of seizures. In a study investigating the timing of seizures in respect to the moon phase using patient data from an epilepsy monitoring unit, a negative correlation was identified between seizures and the fraction of the moon illuminated by the sun. Because these findings disappeared following correction of the clarity of the night, this study suggested that nocturnal light during full moon phases may decrease the frequency of seizures during these times [49].

\section{Seizure Patterns in Epilepsy Syndromes}

Certain epilepsy syndromes may manifest with different seizure patterns. Seizures in juvenile myoclonic epilepsy most frequently occur during the transition from sleep to wakefulness [50]. Studies of seizure patterns in West Syndrome found that seizures occurred more frequently during the morning hours [51]. The characteristic occurrence of early morning idiopathic generalized tonic clonic seizures in some patients is considered as a distinct syndrome [52]. Patients with specific epilepsy syndromes may have more complex seizure cluster patterns that may open an additional therapeutic window.

\section{Epilepsy Modifies the Circadian Rhythm and Sleep}

The relationship between epilepsy and the circadian rhythm is most likely bidirectional. Melatonin levels are on average lower in patients with epilepsy compared with controls [53], and additionally, patients with epilepsy may have stronger fluctuations between the day and nighttime levels of the hormone [54]. Patients with epilepsy are known to have a wide variety of changes in sleep architecture [55•]. These include macrostructural sleep abnormalities, including increased number of awakenings, reduced sleep efficiency, sleep fragmentation, and abnormal K-complexes. Some of these changes may in turn lead to worsening of seizures [56].

\section{Sleep Deprivation and Epilepsy}

There are strong associations between sleep deprivation and seizures. Sleep deprivation is well known, for example, to aggravate seizures in patients with juvenile myoclonic epilepsy and it may also play a role in other forms of epilepsy and related interictal epileptiform discharges. In a retrospective study evaluating the presence of epileptiform discharges among patients undergoing sleep deprivation, $52 \%$ of patients presented with spike activation on EEG following sleep deprivation [57]. Sleep deprivation may also play an important role in patients whose seizures disrupt sleep. Sleep deprivation and modifications of sleep schedules may be used in selected patients in the epilepsy monitoring unit during presurgical workup to be able to inject seizures with radioactive tracer during presurgical ictal Single Photon Emission Computed Tomography (SPECT) evaluations.

\section{Chronopharmacokinetics of Epilepsy Medications}

\section{Overview of Chronopharmacokinetics in Epilepsy}

Endogenous cycles can affect all aspects of the pharmacodynamics and pharmacokinetics of antiepileptic medications. A variety of parameters, including the peak drug plasma concentration $\left(\mathrm{C}_{\max }\right)$, time to reach maximum concentration $\left(t_{\max }\right)$, area under the concentration-time curve (AUC), volume of distribution ( $\mathrm{Vd})$, protein binding, elimination half-life $\left(t_{1 / 2}\right)$, and clearance $(C L)$ can be affected by the timing and delivery of drugs. Rational chronotherapeutic 
strategies require a thorough understanding of how drug pharmacokinetics are affected by their timing and delivery.

Chronopharmacokinetics is the study of changes in the absorption, metabolism, distribution, and elimination of drugs in the body. Absorption may be altered based on dietary cues and circadian gene expression: lipophilic drugs, for example, are absorbed faster in the morning compared with later in the day [58]. Distribution may be affected by cyclic rates of blood flow to various organs and capillary blood flow. Furthermore, free plasma levels of drugs show rhythmic changes [58]. The elimination of drugs can be altered by variations in the rates of metabolism and excretion of drugs over time [58, 59]. The use of specialized formulations, such as galenic drug delivery systems, may alter the digestion and rate of absorption of drugs $[58,60]$ and are an important consideration in formulating a workable chronotherapeutic strategy.

\section{Animal Studies}

Some of the earliest data suggesting an effect of circadian and other cycles affecting the pharmacokinetics of antiepileptic drugs (AEDs) were obtained from animal studies. A variety of components involved in the absorption, distribution, metabolism, and excretion of drugs are likely affected by endogenous rhythms. Variations in plasma protein levels may alter AED availability. Free levels of carbamazepine administered in rodents, for example, are nearly twice as high around noon as compared with the morning. These changes are noted to be inversely proportional to diurnal changes in albumin concentration. Varying protein levels may therefore alter the availability of bioactive drug throughout the day [61].

Changes in plasma levels of drugs may also relate to patterns of drug excretion. In a study evaluating mice continuously infused with valproate via mini-pumps, drug levels were noted to be highest during light exposure. Clearance of drug was greatest during dark exposure [62]. Changes in pharmacokinetic parameters may therefore occur irrespective of the timing of drug administration due to differential excretion of the drug.

\section{Human Studies}

\section{Digestion and Absorption}

The timing of medication in respect to meals plays an important role in the absorption of AEDs. The absorption of valproate, for example, can be delayed for hours if taken after a meal [63]. Consumption of breakfast prior to intake of carbamazepine is noted to delay the absorption of delayed-release forms of the drug disproportionately compared with the standard form [64]. The quantity of food taken at meals may play a role as well. Subjects who take a light breakfast and heavy dinner are more likely to have a higher maximal plasma or serum concentration $\left(\mathrm{C}_{\max }\right)$, shorter time after administration needed to achieve maximal plasma or serum level $\left(\mathrm{t}_{\max }\right)$, and a larger absorption rate $(\mathrm{Ka})$ following morning dose of valproate compared with those who take a light breakfast and light dinner [65].

\section{Distribution}

The distribution of medications is also affected by cyclic changes. Binding to plasma proteins plays an important role in the distribution of valproate: maximal free concentrations of the drug occur between 2-6 AM. In contrast, valproate is most likely to be bound to proteins during the afternoon [66]. Variations in red blood cell concentrations may modify the distribution of valproate, $20 \%$ of which is conveyed in erythrocytes $[67,68]$. In patients taking morning and evening doses of valproate, the drug achieves higher serum concentrations and decreased $t_{\max }$ following the morning dose, in comparison to the evening dose [69].

The distributions of other AEDs likely vary as well in relation to circadian and diurnal rhythms. Mild day/night fluctuations have been observed in patients on continuous infusion of midazolam [70]. In a study with 28 healthy volunteers comparing the pharmacokinetics of diazepam, subjects who took the medication orally demonstrated a more rapid $\mathrm{C}_{\max }$ and shorter $\mathrm{T}_{\max }$ following the morning dose compared with the evening dose. These changes may be accounted for by changes in the absorption and distribution of the medication [71]. Another study noted that plasma levels of carbamazepine and related metabolites were higher 24 hours post-dose during the night compared with the day, a finding which may be due to changes in plasma distribution and protein binding [72]. To date, no other studies have investigated similar changes with other AEDs.

\section{Metabolism}

Selective enzyme expression in the liver may impact the metabolism of drugs. Clock genes in mammalian hepatic cells are largely induced by feeding cycles rather than the suprachiasmatic nucleus [73]. Circadian patterns have been observed in murine glutathione-S-transferase, an enzyme needed for drug conjugation [74, 75]. The CYP3A4 gene, required for digestion of carbamazepine, may be expressed in a diurnal fashion, as demonstrated in an in vitro study [76]. Beta oxidation has also shown diurnal changes in activity [77], and the effect of Beta oxidation on anticonvulsant therapy still under investigation.

\section{Excretion}

Circadian oscillations in renal function may cause changes in glomerular filtration [78] and may impact the excretion of 
certain electrolytes $[79,80]$. Some of these changes may impact the manner in which the kidney excretes medications or metabolites. The excretion of 3-oxo-VPA and VPA glucuronides, metabolites of valproic acid, has been observed to be least between 2-8 AM, a factor that could not be accounted to differences in creatinine excretion [81]. This finding suggests that the excretion of some drugs may be affected by diurnal or circadian patterns.

\section{Other Factors}

Behavioral factors may also play an important role in the timing of dosage of AEDs. People who woke up earlier on free days, or "morning types," tended to take their medications earlier in the day compared with those who woke up later [82•]. Beyond the pharmacokinetic modeling of individual medications, these factors must also be considered when formulating chronotherapeutic treatment strategies.

\section{Chronopharmacodynamics of Epilepsy Medications}

AEDs can also modify circadian, diurnal, or wake/sleep patterns. AEDs may have varying toxicity or efficacy depending on the timing of their administration. These in turn may change the efficacy or duration of AEDs or of intrinsic seizure activity. A number of human and animal studies have explored chronopharmacokinetic aspects of antiepileptic drugs (Table 2).

Animal Studies

\section{Effect of Benzodiazepines on Gene Transcription}

Medications may alter the transcription and activity of circadian-related genes. Diazepam was noted to alter the phase of the circadian clock by increasing or decreasing the expression of clock genes in adult and embryonic zebrafish [83]. This finding may possibly extend to other benzodiazepines.

\section{Timing of Drug Administration and Toxicity of Valproate}

A study investigating such effects in mice entrained by a strict 12-hour light cycle evaluated the toxicity of valproate given at certain times found that mortality was highest when administered at $5 \mathrm{PM}$ and least when administered at $9 \mathrm{PM}$ and $1 \mathrm{PM}$. These findings, which were noted despite a lack of difference in cerebrospinal fluid and plasma concentrations of the drug, suggest that the toxic effects of valproate may be related to the underlying sensitivity of the CNS to the drug at different periods of the day [84]. A murine study found that the valproate was most toxic when administered 17 hours after light onset. Least toxicity occurred when it was administrated 9 hours after light onset, which corresponds to the second half of the night in humans [85•].

\section{Embryotoxicity of AEDs may be Related to Drug Timing}

Teratogenic effects of medications to the fetus may have variations depending on the timing of administration. In a study evaluating effects of valproate given to pregnant mice, the highest fetal mortality occurred when the drug was injected at $5 \mathrm{PM}$. Lower mortality occurred when given at $1 \mathrm{AM}$. These findings may carry important implications in limiting the toxicity of AEDs in women of childbearing age [86].

\section{Human Studies}

\section{Valproate}

Valproate was found to decrease the sensitivity of melatonin secretion to light in patients treated with a single dose for bipolar disorder. This did not have an effect on overall melatonin secretion or the dim-light melatonin onset time [87]. A study evaluating the circadian effects of valproate on Drosophilia found that the drug lengthened the period of the circadian rhythm and promoted rhythmicity [88]. Real-time PCR analysis on mice and human fibroblasts has shown that the valproate can advance or delay the rhythmic expression of the circadian gene Period2::Luciferace depending on the time of administration [89].

\section{Other Medications}

Diazepam may delay the secretion of melatonin, and, according to the above mentioned studies, alter the transcription of circadian genes. Disturbance of sleep architecture has been investigated in carbamazepine, levetiracetam, lamotrigine, vigabatrin and gabapentin [90-92]. It is unclear if and to what extent these drugs modify the sleep/wake cycle or circadian rhythm.

\section{Trials of Chronopharmacotherapy in Epilepsy}

To date, few studies have attempted to utilize principles of chronopharmacology in differential dosing trials. As described above, a number of animal studies have also investigated chronopharmacological aspects of drug toxicity.

Timed Dosing in Patients with Carbamazepine and Phenytoin

Yegnanarayan and colleagues tested the efficacy of differential dosing of carbamazepine and phenytoin in epilepsy patients $[9 \bullet \cdot$. The investigators evaluated 52 patients in 


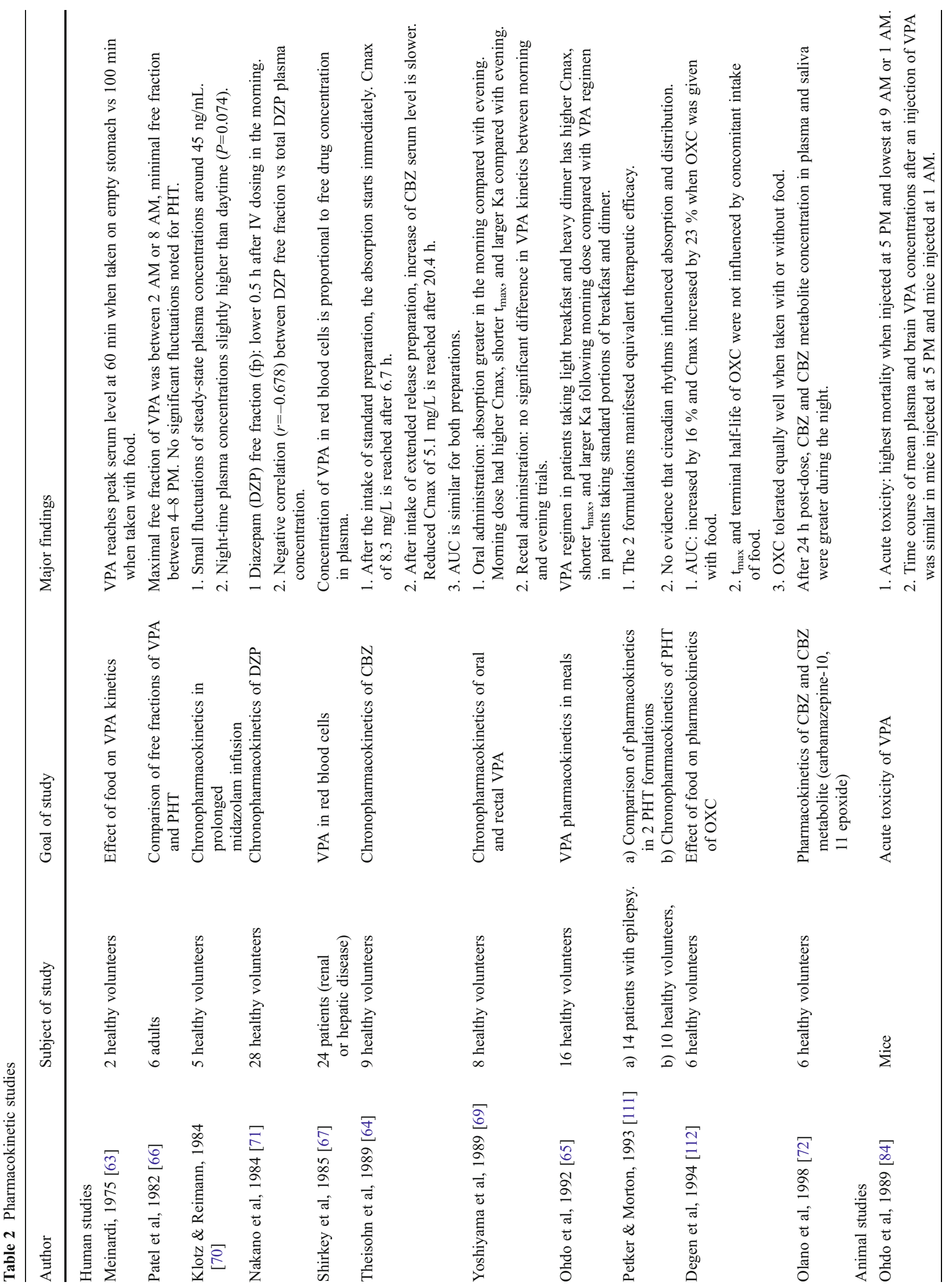


whom the total dose of medication was kept constant but in whom the majority of the dose was given at $8 \mathrm{PM}$. The study found that subjects in the trial group were more likely to reach therapeutic drug levels following 4 weeks of therapy compared with controls. Controls were treated with twice- or once-daily regimens. More patients in the study group attained better seizure control compared with controls.

The investigators focused their study on patients found to have subtherapeutic drug levels on monitoring, and did not study patients specifically in whom seizures followed a specific diurnal pattern. The findings of the study are suggestive of the ability of differential dosing to limit the emergence of drug troughs.

\section{Nocturnal Medication Dosing}

A more targeted approach towards differential dosing was attempted in a pilot trail, published by Guilhoto et al. Eighteen patients with nocturnal or early-morning seizures refractory to conventional AED therapy were prospectively enrolled in a differential dosing trial. Patients were treated with a schedule in which the evening dose was twice the morning dose, keeping the total dose of medication constant. Of the 18 patients treated by this dosing strategy, 11 patients became seizure-free following a mean follow up time of 5.3 months, and 4 patients had a $75 \%-90 \%$ reduction of seizures. This study suggested that chronotherapy can provide improved seizure control compared with conventional therapy in carefully selected patients $[8 \bullet \bullet$.

\section{Limiting Toxic Effects of Antiepileptic Drugs}

Chronopharmacology may provide avenues towards limiting the adverse effects of antiepileptic medications. As a part of one chronotherapy trial, Yegnanarayan evaluated the tolerance the adverse effects of patients treated on a chronotherapeutic schedule. While symptoms of toxicity were present in both groups, symptoms of toxicity subsided more rapidly in patients treated with differential dosing [9••]. In the pilot study conducted by Guilhoto, $2 / 18$ subjects complained of fatigue [8••].

\section{Other Avenues of Chronotherapy in Epilepsy}

Melatonin

Melatonin has been noted to rise following seizures. This change has contributed to reports that indicate a potential anticonvulsant effect of melatonin [93]. Melatonin has been postulated to suppress seizure activity at certain doses. Potential explanations for this effect include antioxidant activity [94] or GABA-potentiating effects [95]. A recent review 
of the literature noted that published trials of melatonin therapy were limited and delivered conflicting results [96]. More research will be needed in order to evaluate the efficacy of melatonin for epilepsy. However, melatonin is also used as chronotherapeutic medication to adjust the circadian rhythm, and may therefore have a role in the alignment of the circadian rhythm [97].

\section{Light Therapy}

Light therapy has been proposed as a seizure management technique. A double-blinded randomized control trial evaluating light therapy in intractable focal epilepsy found that the light therapy may lead to a mild reduction of seizures in these patients. Light therapy may be more useful in patients with specific pathologies, such as hippocampal sclerosis $[98 \cdot]$.

\section{Closed Loop Chronopharmacology}

\section{Seizure Diaries}

Chronotherapy relies on identifying periods of seizure clusters in individual patients. For this, accurate logging of seizures in diaries is needed in order to formulate individualized treatment regimens. The timing of seizures can be self-recorded by seizure diaries to formulate the times of greatest seizure risk and electronic seizure diaries may help [99•]. When seizures of larger groups of patients using these electronic diaries are classified according to their peak hours of occurrence, an early morning peak can be seen in generalized epilepsies, a finding that is consistent with the existing literature on seizure patterns [99•]. Seizure diaries may thus be useful in identifying the timing of seizure clusters, which in turn is an important factor in determining dosing strategies.

\section{Automated Seizure Detection Systems}

Seizure diaries are an important means towards establishing individual seizure patterns. However, they present with a number of shortcomings. Patients may not log all seizures, and they may not be aware of all epileptic events. In view of this, automated systems may provide more detailed data relating to the timing of seizures. Such systems, currently under investigation, include actigraphy, the use of electrodermal sensors to monitor sweat secretion or continuous electrocorticography among others $[100 \bullet, 101 \bullet]$. These sensors may allow for continuous data collection and seizure monitoring. Identification of specific diurnal patterns may enable clinicians to tailor treatment towards individualized chronotype patterns.
Closed Loop Treatments

Automated seizure detection methods may allow for seizure warning and abortion prior to clinical seizure onset. Closed loop systems including a seizure detector and auto-corrective mechanisms have been designed for epilepsy. To date, the studied systems, such as the responsive neurostimulation device developed by Neuropace, Inc. (Mountain View, California, USA) have utilized localized neuromodulation techniques to abort imminent seizures [100•]. The use of localized medications may also be a useful way to abort or minimize seizure activity. In murine experiments, intra-hippocampal adenosine was shown to reduce seizure frequency [102]. Continuous intra-amygdala and intra-thalamic infusion of gamma amino butyric acid has been similarly shown to reduce kindling [103]. Closed loop methods utilizing these types of local drug delivery systems may serve as useful techniques for seizure abortion. These localized delivery systems in combination with individual timing of medication according to patterns of seizures may allow for highly targeted and specific therapy in line with endogenous rhythms (Fig. 1).

\section{Conclusions}

Chronotherapy carries potential to better control seizures and limit adverse effects. More data are needed to study the manner in which endogenous rhythms alter the digestion, absorption, distribution, metabolism and excretion of AEDs. In addition, antiepileptic drugs may themselves cause changes in circadian patterns in ways that are currently poorly understood. Pharmacokinetic and phamacodynamic profiles of individual medications need to be clearly elucidated.

Chronotherapy has important clinical implications for epilepsy management. Patients are encouraged to track the timing of their seizures, potentially using electronic seizure detection devices. Seizure diaries may be especially useful for this purpose, and some electronic diaries now also provide a breakdown of seizure timing and thereby assist in medication management. We are currently working on algorithms that can automatically implement this clinical information in outcome prediction and treatment algorithms and these electronic aids may therefore help clinicians with treatment choices in the future. Furthermore, differential dosing strategies or temporary treatment with intermittent antiepileptic medication therapy may be feasible in patients with well-defined seizure clusters. Clinicians should be aware that this approach has not yet been thoroughly studied and that complications including increased toxicity and breakthrough seizures may occur. While these caveats should be considered, the clinical applications of chronopharmacology may lead to improved seizure control in some patients. 
In order for chronotherapy to have a firm footing in clinical practice, more data are needed to evaluate which patients may benefit from chronotherapy. Further study is also needed to formulate dosing strategies based on patientspecific seizure patterns. Future study should be based on retrospective reviews, bimarker studies, and surveys. Additionally, randomized controlled trials should be performed in the next few years. In addition to treatment with traditional AEDs, novel avenues of chronotherapy include melatonin and light therapy. While more data are needed, the available evidence suggests that chronotherapy may play an important role in providing better control of seizures.

Disclosures Sriram Ramgopal declares no conflict of interest. Sigride Thome-Souza is a recipient of the CNPq scholarship (246205/2012-1) from the Brazilian government. Tobias Loddenkemper serves on the Laboratory Accreditation Board for Long Term (Epilepsy and Intensive Care Unit) Monitoring, on the Council of the American Clinical Neurophysiology Society, on the American Board of Clinical Neurophysiology, as an Associate Editor for Seizure, and performs video electroencephalogram long-term monitoring, electroencephalograms, and other electrophysiological studies at Boston Children's Hospital and bills for these procedures. He receives support from the National Institutes of Health/NINDS, a Career Development Fellowship Award from Harvard Medical School and Boston Children's Hospital, the Program for Quality and Safety at Boston Children's Hospital, the Payer Provider Quality Initiative, The Epilepsy Foundation of America (EF-213583 and EF-213882), the Center for Integration of Medicine and Innovative Technology, the Epilepsy Therapy Project, the American Epilepsy Society, Cure, the Pediatric Epilepsy Research Foundation, and investigator initiated research grants from Lundbeck and Eisai.

Open Access This article is distributed under the terms of the Creative Commons Attribution License which permits any use, distribution, and reproduction in any medium, provided the original author(s) and the source are credited.

\section{References}

Papers of particular interest, published recently, have been highlighted as:

- Of importance

•- Of major importance

1. MacDonald BK, Johnson AL, Goodridge DM, Cockerell OC, Sander JW, Shorvon SD. Factors predicting prognosis of epilepsy after presentation with seizures. Ann Neurol. 2000;48:833-41.

2. Kahane P. Epilepsy surgery in adult patients: for whom? Rev Neurol (Paris). 2004;160:5S179-84.

3. Liu L, Henry JC. New-onset partial epilepsy in adults. Curr Treat Options Neurol. 2009;11:242-52.

4. Uthman BM. Vagus nerve stimulation for seizures. Arch Med Res. 2000;31:300-3.

5. - Durazzo TS, Spencer SS, Duckrow RB, Novotny EJ, Spencer DD, Zaveri HP. Temporal distributions of seizure occurrence from various epileptogenic regions. Neurology. 2008;70:1265-71. This study evaluates the diurnal occurrence of seizures in an adult series in which epilepsy was sufficiently localized for surgical resection.

6. Loddenkemper T, Vendrame M, Zarowski M, Gregas M, Alexopoulos AV, Wyllie E, et al. Circadian patterns of pediatric seizures. Neurology. 2011;76:145-53.

7. • Pavlova MK, Woo Lee J, Yilmaz F, Dworetzky BA. Diurnal pattern of seizures outside the hospital: is there a time of circadian vulnerability? Neurology. 2012;78:1488-92. This study evaluates diurnal seizure patterns in patients undergoing ambulatory EEG.

8. •- Guilhoto LM, Loddenkemper T, Vendrame M, Bergin A, Bourgeois BF, Kothare SV. Higher evening antiepileptic drug dose for nocturnal and early-morning seizures. Epilepsy Behav. 2011;20:334-7. This is a differential dosing strategy in epilepsy reported to date.Uses patients with refractory epilepsy with nocturnal or early morning seizure clusters.

9. •• Yegnanarayan R, Mahesh SD, Sangle S. Chronotherapeutic dose schedule of phenytoin and carbamazepine in epileptic patients. Chronobiol Int. 2006;23:1035-46. This is one of the earliest trials of differential dosing strategy in epilepsy and is the largest published cohort to date.Used patients with subtherapeutic drug troughs.

10. Hofstra WA, de Weerd AW. How to assess circadian rhythm in humans: a review of literature. Epilepsy Behav. 2008;13:438-44.

11. Molina TA, Burgess HJ. Calculating the dim light melatonin onset: the impact of threshold and sampling rate. Chronobiol Int. 2011;28:714-8.

12. Sharkey KM, Carskadon MA, Figueiro MG, Zhu Y, Rea MS Effects of an advanced sleep schedule and morning short wavelength light exposure on circadian phase in young adults with late sleep schedules. Sleep Med. 2011;12:685-92.

13. Shekleton JA, Parcell DL, Redman JR, Phipps-Nelson J, Ponsford JL, Rajaratnam SM. Sleep disturbance and melatonin levels following traumatic brain injury. Neurology. 2010;74:1732-8.

14. Conroy DA, Hairston IS, Arnedt JT, Hoffmann RF, Armitage R, Brower KJ. Dim light melatonin onset in alcohol-dependent men and women compared with healthy controls. Chronobiol Int. 2012;29:35-42.

15. Hofstra WA, Gordijn MCM, van der Palen J, van Regteren R, Grootemarsink BE, de Weerd AW. Timing of temporal and frontal seizures in relation to the circadian phase: a prospective pilot study. Epilepsy Res. 2011;94:158-62.

16. Waterhouse J, Weinert D, Minors D, Folkard S, Owens D, Atkinson $\mathrm{G}$, et al. A comparison of some different methods for purifying core temperature data from humans. Chronobiol Int. 2000;17:539-66.

17. Dijk DJ, Czeisler CA. Paradoxical timing of the circadian rhythm of sleep propensity serves to consolidate sleep and wakefulness in humans. Neurosci Lett. 1994;166:63-8.

18. Youan BB. Chronopharmaceutics: gimmick or clinically relevant approach to drug delivery? J Control Release. 2004;98:337-53.

19. - Singh R, Sharma PK, Malviya R. Circadian cycle and chronotherapeutics: recent trend for the treatment of various biological disorders. Recent Pat Drug Deliv Formul. 2012;6:80-91. This review discusses the potential uses, and various dosing preparations for chronopharmacology.

20. Acelajado MC, Pisoni R, Dudenbostel T, Oparil S, Calhoun DA, Glasser SP. Both morning and evening dosing of nebivolol reduces trough mean blood pressure surge in hypertensive patients. J Am Soc Hypertens. 2012;6:66-72.

21. Kenny AM, Schneider MB, Baden DR, Pfeiffer RF, Murrin LC. Bromocriptine holiday: effects on dopamine receptors and turning behavior in rats. Neurology. 1986;36:400-4.

22. Sato Y, Kobayashi E, Hakamata Y, Kobahashi M, Wainai T, Murayama T, et al. Chronopharmacological studies of ketamine in normal and NMDA epsilon1 receptor knockout mice. Br J Anaesth. 2004;92:859-64. 
23. Tekade AR, Gattani SG. Dual cross-linked pulsatile beads for chronotherapy of asthma: development and evaluation. Drug Deliv. 2010;17:581-6.

24. Tsioufis C, Kasiakogias A, Thomopoulos C, Stefanadis C. Nighttime blood pressure in diabetes: better treat it than leave it. Am J Hypertens. 2012;25:279.

25. Shea SA. Obesity and pharmacologic control of the body clock. N Engl J Med. 2012;367:175-8.

26. Etienne-Grimaldi MC, Cardot JM, Francois E, Renee N, Douillard JY, Gamelin E, et al. Chronopharmacokinetics of oral tegafur and uracil in colorectal cancer patients. Clin Pharmacol Ther. 2008;83:413-5.

27. Portaluppi F, Hermida RC. Circadian rhythms in cardiac arrhythmias and opportunities for their chronotherapy. Adv Drug Deliv Rev. 2007;59:940-51.

28. Ezeugo U, Glasser SP. Clinical benefits vs shortcomings of diltiazem once-daily in the chronotherapy of cardiovascular diseases. Expert Opin Pharmacother. 2009;10:485-91.

29. Lieverse R, der Lek RF R-v, Voermans JM, Hoogendijk WJ. Chronotherapy for affective disorders. Tijdschr Psychiatr. 2012; 54:527-37.

30. Karwa R, Shashank A, Rambhau D, Gopinath D, Ravi D. Timedependent pharmacokinetic interaction between zidovudine and rifampicin following oral administration at 10.00 and 22.00 hours. Drug Metabol Drug Interact. 2001;18:123-34.

31. King AB, Clark D, Wolfe GS. How much do I give? Dose estimation formulas for once-nightly insulin glargine and premeal insulin lispro in type 1 diabetes mellitus. Endocr Pract. 2012;18:382-6.

32. Gowers, W. Epilepsy and other chronic convulsive diseases: their causes, symptoms and treatment. New York: William Wood; 1885.

33. Manfredini R, Vergine G, Boari B, Faggioli R, Borgna-Pignatti C. Circadian and seasonal variation of first febrile seizures. J Pediatr. 2004;145:838-9.

34. Cortez MA, Burnham WM, Hwang PA. Infantile spasms: seasonal onset differences and zeitgebers. Pediatr Neurol. 1997;16:220-4.

35. Misra UK, Kalita J. Overview: Japanese encephalitis. Prog Neurobiol. 2010;91:108-20.

36. Hofstra WA, Grootemarsink BE, Dieker R, van der Palen J, de Weerd AW. Temporal distribution of clinical seizures over the 24 hday: a retrospective observational study in a tertiary epilepsy clinic. Epilepsia. 2009;50:2019-26. This pilot study is the first to associate seizures to a circadian biomarker, namely dim-light melatonin onset.

37. Pavlova MK, Shea SA, Bromfield EB. Day/night patterns of focal seizures. Epilepsy Behav. 2004;5(1):44-9.

38. Karafin M, St Louis EK, Zimmerman MB, Sparks JD, Granner MA. Bimodal ultradian seizure periodicity in human mesial temporal lobe epilepsy. Seizure. 2010;19:347-51.

39. - Zarowski M, Loddenkemper T, Vendrame M, Alexopoulos AV, Wyllie E, Kothare SV. Circadian distribution and sleep/wake patterns of generalized seizures in children. Epilepsia. 2011;52:107683. This article discusses the importance of age considerations in seizure timing and is the first to consider how these factors may change with time.

40. Ramgopal S, Shah A, Zarowski M, Vendrame M, Gregas M, Alexopoulos AV, et al. Diurnal and sleep/wake patterns of epileptic spasms in different age groups. Epilepsia. 2012;53:1170-7.

41. Ramgopal S, Vendrame M, Shah A, Gregas M, Zarowski M, Rotenberg A, et al. Circadian patterns of generalized tonic-clonic evolutions in pediatric epilepsy patients. Seizure. 2012;21:535-9.

42. Sánchez Fernández I, Ramgopal S, Powell C, Gregas M, Zarowski M, Shah A, et al. Clinical evolution of seizures: distribution across time of day and sleep/wakefulness cycle. J Neurol. 2012:26:54957.

43. Nobili L, Cardinale F, Magliola U, Cicolin A, Didato G, Bramerio $\mathrm{M}$, et al. Taylor's focal cortical dysplasia increases the risk of sleeprelated epilepsy. Epilepsia. 2009;50:2599-604.
44. Crespel A, Baldy-Moulinier M, Coubes P. The relationship between sleep and epilepsy in frontal and temporal lobe epilepsies: practical and physiopathologic considerations. Epilepsia. 1998; 39:150-7.

45. Herman ST, Walczak TS, Bazil CW. Distribution of partial seizures during the sleep-wake cycle: differences by seizure onset site. Neurology. 2001;56:1453-9.

46. Sinha S, Brady M, Scott CA, Walker MC. Do seizures in patients with refractory epilepsy vary between wakefulness and sleep? J Neurol Neurosurg Psychiatry. 2006;77:1076-8.

47. Herzog AG, Klein P, Ransil BJ. Three patterns of catamenial epilepsy. Epilepsia. 1997;38:1082-8.

48. Foldvary-Schaefer N, Harden C, Herzog A, Falcone T. Hormones and seizures. Cleve Clin J Med. 2004;71 Suppl 2:S11-8.

49. Baxendale S, Fisher J. Moonstruck? The effect of the lunar cycle on seizures. Epilepsy Behav. 2008;13:549-50.

50. Panayiotopoulos CP, Obeid T, Tahan AR. Juvenile myoclonic epilepsy: a 5-year prospective study. Epilepsia. 1994;35:285-96.

51. Ruiz-Miyares F, Siegmund R, Wermke K, Dorado-Gallego J, Escobedo-Beceiro DI. West syndrome: a chronobiological approach. Rev Neurol. 2000;30:925-8.

52. Engel Jr J. A proposed diagnostic scheme for people with epileptic seizures and with epilepsy: report of the ILAE Task Force on Classification and Terminology. Epilepsia. 2001;42:796-803.

53. Yalyn O, Arman F, Erdogan F, Kula M. A comparison of the circadian rhythms and the levels of melatonin in patients with diurnal and nocturnal complex partial seizures. Epilepsy Behav. 2006;8:542-6.

54. Molina-Carballo A, Munoz-Hoyos A, Rodriguez-Cabezas T, Acuna-Castroviejo D. Day-night variations in melatonin secretion by the pineal gland during febrile and epileptic convulsions in children. Psychiatry Res. 1994;52:273-83.

55. - Larson AM, Ryther RC, Jennesson M, Geffrey AL, Bruno PL, Anagnos CJ, et al. Impact of pediatric epilepsy on sleep patterns and behaviors in children and parents. Epilepsia. 2012;53:1162-9. Excellent review documenting the effects of epilepsy on sleep patterns.

56. Kothare SV, Zarowski M. Sleep and epilepsy: common bedfellows. J Clin Neurophysiol. 2011;28:101-2.

57. Fountain NB, Kim JS, Lee SI. Sleep deprivation activates epileptiform discharges independent of the activating effects of sleep. J Clin Neurophysiol. 1998;15:69-75.

58. Bruguerolle B. Chronopharmacokinetics. Current status. Clin Pharmacokinet. 1998;35:83-94.

59. Baraldo M. The influence of circadian rhythms on the kinetics of drugs in humans. Expert Opin Drug Metab Toxicol. 2008;4:17592.

60. Ohdo S. Chronopharmacology focused on biological clock. Drug Metab Pharmacokinet. 2007;22:3-14.

61. Labrecque G, Belanger PM. Biological rhythms in the absorption, distribution, metabolism, and excretion of drugs. Pharmacol Ther. 1991;52:95-107.

62. Ohdo S, Nakano S, Ogawa N. Chronopharmacokinetics of valproic acid following constant-rate administration in mice. Chronobiol Int. 1991;8:35-43.

63. Meinardi H, Van Der Kleijn E, Meijer JW, Van Rees H. Absorption and distribution of antiepileptic drugs. Epilepsia. 1975;16:353-65.

64. Theisohn M, Assion HJ, Werner U. Influence of breakfast and day time on the disposition of carbamazepine in healthy volunteers after intake of single dose $(600 \mathrm{mg})$ of a standard or retard preparation of carbamazepine. Chronopharmacology in Therapy of the Epilepsies. New York: Raven Press; 1989. p. 31-54.

65. Ohdo S, Nakano S, Ogawa N. Circadian changes of valproate kinetics depending on meal condition in humans. J Clin Pharmacol. 1992;32:822-6. 
66. Patel IH, Venkataramanan R, Levy RH, Viswanathan CT, Ojemann LM. Diurnal oscillations in plasma protein binding of valproic acid. Epilepsia. 1982;23:283-90.

67. Shirkey RJ, Jellett LB, Kappatos DC, Maling TJ, Macdonald A. Distribution of sodium valproate in normal whole blood and in blood from patients with renal or hepatic disease. Eur J Clin Pharmacol. 1985;28:447-52.

68. Oishi K, Ohkura N, Kadota K, Kasamatsu M, Shibusawa K, Matsuda $\mathrm{J}$, et al. Clock mutation affects circadian regulation of circulating blood cells. J Circadian Rhythms. 2006;4:13.

69. Yoshiyama Y, Nakano S, Ogawa N. Chronopharmacokinetic study of valproic acid in man: comparison of oral and rectal administration. J Clin Pharmacol. 1989;29:1048-52.

70. Klotz U, Reimann IW. Chronopharmacokinetic study with prolonged infusion of midazolam. Clin Pharmacokinet. 1984;9:469-74.

71. Nakano S, Watanabe H, Nagai K, Ogawa N. Circadian stagedependent changes in diazepam kinetics. Clin Pharmacol Ther. 1984;36:271-7.

72. Olano I, Vázquez M, Fagiolino P. Chronopharmacocinétique de la carbamazépine et de son métabolite 10.11 époxide. Journal de Pharmacie Clinique. 1998;17:153-6.

73. Stokkan KA, Yamazaki S, Tei H, Sakaki Y, Menaker M. Entrainment of the circadian clock in the liver by feeding. Science. 2001;291:490-3.

74. Tunon MJ, Gonzalez P, Lopez P, Salido GM, Madrid JA. Circadian rhythms in glutathione and glutathione-S transferase activity of rat liver. Arch Int Physiol Biochim Biophys. 1992;100:83-7.

75. Jaeschke H, Wendel A. Diurnal fluctuation and pharmacological alteration of mouse organ glutathione content. Biochem Pharmacol. 1985;34:1029-33.

76. Takiguchi T, Tomita M, Matsunaga N, Nakagawa H, Koyanagi S, Ohdo S. Molecular basis for rhythmic expression of CYP3A4 in serum-shocked HepG2 cells. Pharmacogenet Genomics. 2007; 17:1047-56.

77. Fuse Y, Hirao A, Kuroda H, Otsuka M, Tahara Y, Shibata S. Differential roles of breakfast only (1 meal per day) and a bigger breakfast with a small dinner ( 2 meals per day) in mice fed a highfat diet with regard to induced obesity and lipid metabolism. J Circadian Rhythms. 2012;10:4.

78. Vagnucci AH, Shapiro AP, McDonald Jr RH. Effects of upright posture on renal electrolyte cycles. J Appl Physiol. 1969;26:720-31.

79. Gumz ML, Cheng KY, Lynch IJ, Stow LR, Greenlee MM, Cain $\mathrm{BD}$, et al. Regulation of alphaENaC expression by the circadian clock protein Period 1 in mpkCCD(c14) cells. Biochim Biophys Acta. 2010;1799:622-9.

80. Min HK, Jones JE, Flink EB. Circadian variations in renal excretion of magnesium, calcium, phosphorus, sodium, and potassium during frequent feeding and fasting. Fed Proc. 1966;25:917-21.

81. Reith DM, Andrews J, McLaughlin D. Valproic acid has temporal variability in urinary clearance of metabolites. Chronobiol Int. 2001;18:123-9.

82. - Hofstra WA, van der Palen J, de Weerd AW. Morningness and eveningness: when do patients take their antiepileptic drugs? Epilepsy Behav. 2012;23:320-3. Excellent study evaluating the effect of behavioral/chronotype patterns in the timing of drug administration.

83. Oggier DM, Weisbrod CJ, Stoller AM, Zenker AK, Fent K. Effects of diazepam on gene expression and link to physiological effects in different life stages in zebrafish Danio rerio. Environ Sci Technol. 2010;44:7685-91.

84. Ohdo S, Nakano S, Ogawa N. Chronotoxicity of sodium valproate and its mechanisms in mice: dose-concentration-response relationship. Chronobiol Int. 1989;6:229-35.

85. - Ben-Cherif W, Dridi I, Aouam K, Ben-Attia M, Reinberg A, Boughattas NA. Chronotolerance study of the antiepileptic drug valproic acid in mice. J Circadian Rhythms. 2012;10:3. Excellent study evaluating variation in toxicity of valproate based on the timing of drug administration.
86. Ohdo S, Watanabe H, Ogawa N, Yoshiyama Y, Sugiyama T. Chronotoxicity of sodium valproate in pregnant mouse and embryo. Jpn J Pharmacol. 1996;70:253-8.

87. Hallam KT, Olver JS, Norman TR. Effect of sodium valproate on nocturnal melatonin sensitivity to light in healthy volunteers. Neuropsychopharmacology. 2005;30:1400-4.

88. Dokucu ME, Yu L, Taghert PH. Lithium- and valproate-induced alterations in circadian locomotor behavior in Drosophila. Neuropsychopharmacology. 2005;30:2216-24.

89. Johansson AS, Brask J, Owe-Larsson B, Hetta J, Lundkvist GB. Valproic acid phase shifts the rhythmic expression of Period2:: Luciferace. J Biol Rhythms. 2011;26:541-51.

90. Cicolin A, Magliola U, Giordano A, Terreni A, Bucca C, Mutani R. Effects of levetiracetam on nocturnal sleep and daytime vigilance in healthy volunteers. Epilepsia. 2006;47:82-5.

91. Placidi F, Diomedi M, Scalise A, Marciani MG, Romigi A, Gigli GL. Effect of anticonvulsants on nocturnal sleep in epilepsy. Neurology. 2000;54 Suppl 1:S25-32.

92. Raol YH, Meti BL. Effects of vigabatrin on sleep-wakefulness cycle in amygdala-kindled rats. Epilepsia. 2000;41:128-31.

93. Molina-Carballo A, Munoz-Hoyos A, Sanchez-Forte M, UberosFernandez J, Moreno-Madrid F, Acuna-Castroviejo D. Melatonin increases following convulsive seizures may be related to its anticonvulsant properties at physiological concentrations. Neuropediatrics. 2007;38:122-5.

94. Kabuto H, Yokoi I, Ogawa N. Melatonin inhibits iron-induced epileptic discharges in rats by suppressing peroxidation. Epilepsia. 1998;39:237-43.

95. Niles LP, Pickering DS, Arciszewski MA. Effects of chronic melatonin administration on GABA and diazepam binding in rat brain. J Neural Transm. 1987;70:117-24.

96. Brigo F, Del Felice A. Melatonin as add-on treatment for epilepsy. Cochrane Database Syst Rev. 2012;6:CD006967.

97. Sanchez-Barcelo EJ, Mediavilla MD, Reiter RJ. Clinical uses of melatonin in pediatrics. Int J Pediatr. 2011;2011:892624.

98. • Baxendale S, O'Sullivan J, Heaney D. Bright light therapy as an add on treatment for medically intractable epilepsy. Epilepsy Behav. 2012;24:359-64. This is the first published study evaluating light therapy for treatment of epilepsy.

99. - Le S, Shafer PO, Bartfeld E, Fisher RS. An online diary for tracking epilepsy. Epilepsy Behav. 2011;22:705-9. This study evaluates the timing of seizures gathered from a cohort of patients utilizing an online seizure tracking diary system.

100. • Morrell MJ. Responsive cortical stimulation for the treatment of medically intractable partial epilepsy. Neurology. 2011;77:1295304. Reports the results of an implemented closed loop trial for the treatment of epilepsy.

101. • Poh MZ, Loddenkemper T, Reinsberger C, Swenson NC, Goyal $\mathrm{S}$, Sabtala MC, et al. Convulsive seizure detection using a wristworn electrodermal activity and accelerometry biosensor. Epilepsia. 2012;53:e93-7. This study evalutes the use of a wristband which measures electrodermal conductance and accelerometry as a portable seizure detector.

102. Van Dycke A, Raedt R, Dauwe I, Sante T, Wyckhuys T, Meurs A, et al. Continuous local intrahippocampal delivery of adenosine reduces seizure frequency in rats with spontaneous seizures. Epilepsia. 2010;51:1721-8.

103. Barcia JA, Gallego JM. Intraventricular and intracerebral delivery of anti-epileptic drugs in the kindling model. Neurotherapeutics. 2009;6:337-43.

104. Bazil CW, Walczak TS. Effects of sleep and sleep stage on epileptic and nonepileptic seizures. Epilepsia. 1997;38:56-62.

105. Quigg M, Straume M, Menaker M, Bertram 3rd EH. Temporal distribution of partial seizures: comparison of an animal model with human partial epilepsy. Ann Neurol. 1998;43: $748-55$. 
106. Quigg M, Straume M. Dual epileptic foci in a single patient express distinct temporal patterns dependent on limbic vs nonlimbic brain location. Ann Neurol. 2000;48:117-20.

107. Hofstra WA, Spetgens WP, Leijten FS, van Rijen PC, Gosselaar $\mathrm{P}$, van der Palen J, et al. Diurnal rhythms in seizures detected by intracranial electrocorticographic monitoring: an observational study. Epilepsy Behav. 2009;14:617-21.

108. Kaleyias J, Loddenkemper T, Vendrame M, Das R, Syed TU, Alexopoulos AV, et al. Sleep-wake patterns of seizures in children with lesional epilepsy. Pediatr Neurol. 2011;45:109-13.

109. Sanchez Fernandez I, Ramgopal S, Powell C, Gregas M, Zarowski M, Shah A, et al. Clinical evolution of seizures: distribution across time of day and sleep/wakefulness cycle. J Neurol. 2012;260:549 57.

110. Yildiz FG, Tezer FI, Saygi S. Temporal relationship between awakening and seizure onset in nocturnal partial seizures. J Neurol Sci. 2012;315:33-8.

111. Petker MA, Morton DJ. Comparison of the effectiveness of 2 oral phenytoin products and chronopharmacokinetics of phenytoin. J Clin Pharm Ther. 1993;18:213-7.

112. Degen PH, Flesch G, Cardot JM, Czendlik C, Dieterle W. The influence of food on the disposition of the antiepileptic oxcarbazepine and its major metabolites in healthy volunteers. Biopharm Drug Dispos. 1994;15:519-26. 